\title{
Effect of respiratory obstruction on the arterial and venous circulation in animals and man
}

\author{
V. E. NEGUS ${ }^{1}, S$. ORA M ${ }^{2}$, D. C. B A N K S ${ }^{2}$ \\ Department of Physiology, Royal College of Surgeons of England ${ }^{1}$, and Cardiac Department, \\ King's College Hospital, London, S.E.5²
}

\begin{abstract}
The glottis of man, which widens on inspiration, is a choked point on the airway, its lumen even when maximally open being less than that of the trachea. The glottis, however, is not the only point of obstruction, the nose being usually of greater significance, especially in carnivorous species. The nose does not change in calibre during respiration. Experiments were carried out in animals and man, consisting of measurements of intra-tracheal, intra-thoracic, femoral arterial, and right atrial pressures, to determine the effects of normal nasal obstruction of constant presence, increase in nasal resistance, rhythmic obstruction at the glottis, combined nasal and glottic obstruction, and of elimination of nasal resistance and elimination of glottic resistance. As a result of these experiments the early opinion that the pump action on the circulation was due mainly to obstruction at the glottis has proved incorrect, and the main point of resistance is the nose, which offers constant resistance, variable in wide periods of time but not so rapidly as the phases of respiration. It appears that the glottis is the point of fine adjustment.
\end{abstract}

A phenomenon which has interested one of us (V.E. N.) for more than 50 years is the rhythmic changes in calibre of the glottis. These changes are readily observed and are synchronous with the phases of respiration.

During inspiration the glottis widens to a lesser or greater extent, reaching the maximum possible during forced inspiration; on expiration there is narrowing. These movements were described by Semon and others in 1890 or earlier (Negus, 1929b).

The glottis of man is a choked point on the airway, its lumen even when maximally open being less than that of the trachea. Consequently, there is always some obstruction to the passage of air, depending on the degree of glottic opening. Most mammals have a relatively larger and wider glottis than man.

During inspiration descent of the diaphragm and expansion of the thorax draw air through the upper air passages, and of necessity the intratracheal and intra-thoracic pressures are reduced below their resting levels; the reverse occurs during expiration. With an airway of constant calibre some changes in intra-thoracic pressure would occur; but the problem is to discover the reason for the glottic movements, which are considerable.

The changes in intra-thoracic pressure influence the flow of blood into the right side of the heart and its output from the left ventricle.
The effect of deep inspiration in man is shown in Figure 1. Reduced intra-thoracic pressure on inspiration will have a suction effect, causing increased flow of blood into the right atrium through the great veins; it will also lead to dilatation of the pulmonary capillaries, with lowered resistance to the flow of blood through the lungs and thence into the left atrium and ventricle. As can be seen in Fig. 1, on inspiration the pressure rises in the right atrium and this, although not directly related, implies an increase in pulmonary arterial flow.

This increase in pulmonary flow occurs at the time when incoming air reaches the alveoli; the greater volume of blood meets a greater volume of air, with the possibility of increase in the exchange of oxygen and carbon dioxide. This correlation is of obvious physiological advantage.

The converse happens during expiration. The increase in intra-thoracic pressure during expiration has a retarding effect on flow of blood through the superior and inferior venae cavae and also through the thin-walled right atrium; in consequence less blood passes through the narrowed pulmonary capillaries. This is at a time when there is no fresh air in the alveoli and therefore a reduced exchange of oxygen and $\mathrm{CO}_{2}$; here again there is a correlation of physiological advantage.

The extent of these inspiratory and expiratory variations is controlled in part by the degree of opening of the glottis, this aperture thus acting as 


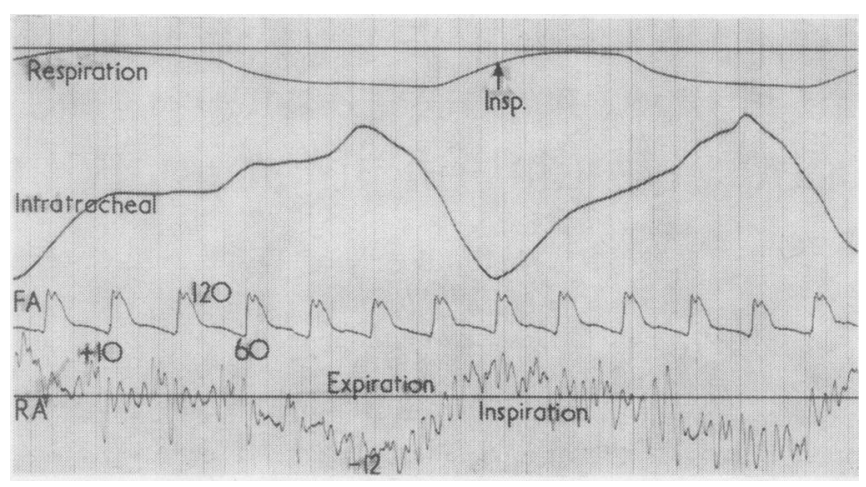

FIG. 1. Human record, showing effects of deep inspiration with nose open and mouth closed. Respiratory movements indicated by a thermistor at the mouth; upstroke is inspiratory (upper curve). Intra-thoracic pressure is recorded through a needle in the trachea, there being a dip during the early part of inspiration (2nd line). Pressure in the aortic system was taken through a needle passed into the femoral artery $(F A)$, but in this case no respiratory waves are recorded. The lowest line $(R A)$ shows the right atrial pressure taken through a catheter passed under radioscopic guidance through a vein in the right arm.

Big inspiratory changes of pressure in the venous system are shown in the right atrial line as waves rising during inspiration to +10 $\mathrm{mm}$. $\mathrm{Hg}$ and dropping during the phase of deep expiration to $-12 \mathrm{~mm}$. $\mathrm{Hg}$, a swing of $22 \mathrm{~mm}$. $\mathrm{Hg}\left(286 \mathrm{~mm}\right.$. $\left.\mathrm{H}_{2} \mathrm{O}\right)$.

a regulator of gases in the lungs; it is also dependent on the degree of resistance to entry of air in the upper air passages.

This is the theoretical explanation of the glottic changes, but confirmatory evidence was essential ; this became possible in 1921 when experiments were carried out by Professor R. J. S. McDowall and one of us (V.E.N.) at King's College, London.

Kymograph tracings showed the respiratory waves on the aortic and pulmonary circulation, varying with the degree of glottic obstruction; some of these tracings were included in The Mechanism of the Larynx (Negus, 1929b), but at the time when they were taken there were no electronic methods of recording. Incidentally it was noticed from the beginning of these investigations that in the cat opening of the glottis is slightly in advance of inspiratory movement of the diaphragm and chest wall, whilst expiratory closure is a little ahead of the movements of chest and thorax.

It was realized that this correlation of air entry and blood flow was not directed mainly to the supply of oxygen, as was at first imagined, but had the more important object of regulating the exchange of $\mathrm{CO}_{2}$, as first suggested by Professor
McDowall. 'A gradually decreasing volume of $\mathrm{CO}_{2}$ is washed out of a gradually decreasing volume of blood, and the ratio between them seems to be maintained by the degree of closure of the glottis. The mechanism is the reverse of that occurring during inspiration' (The Mechanism of the Larynx, p. 161).

It is well recognized that there is usually a considerable excess of oxygen available, well above the needs of the body, but the percentage of carbon dioxide must be kept constant, since it is the main regulator of respiratory exchange (Haldane, 1922).

On subsequent consideration, particularly after the acquisition of knowledge of nasal anatomy in man and animals (Negus, 1958), it was realized that the glottis is not the only point of obstruction on the airway; the nose is usually of greater significance, especially in carnivorous species (Fig. 2 ). The nose does not change in calibre during the phases of respiration, and consequently it is to be looked on as giving a more or less constant obstruction to the inward and outward flow of air ; the glottis appears to supply the fine adjustment, with variations during inspiration and expiration. 


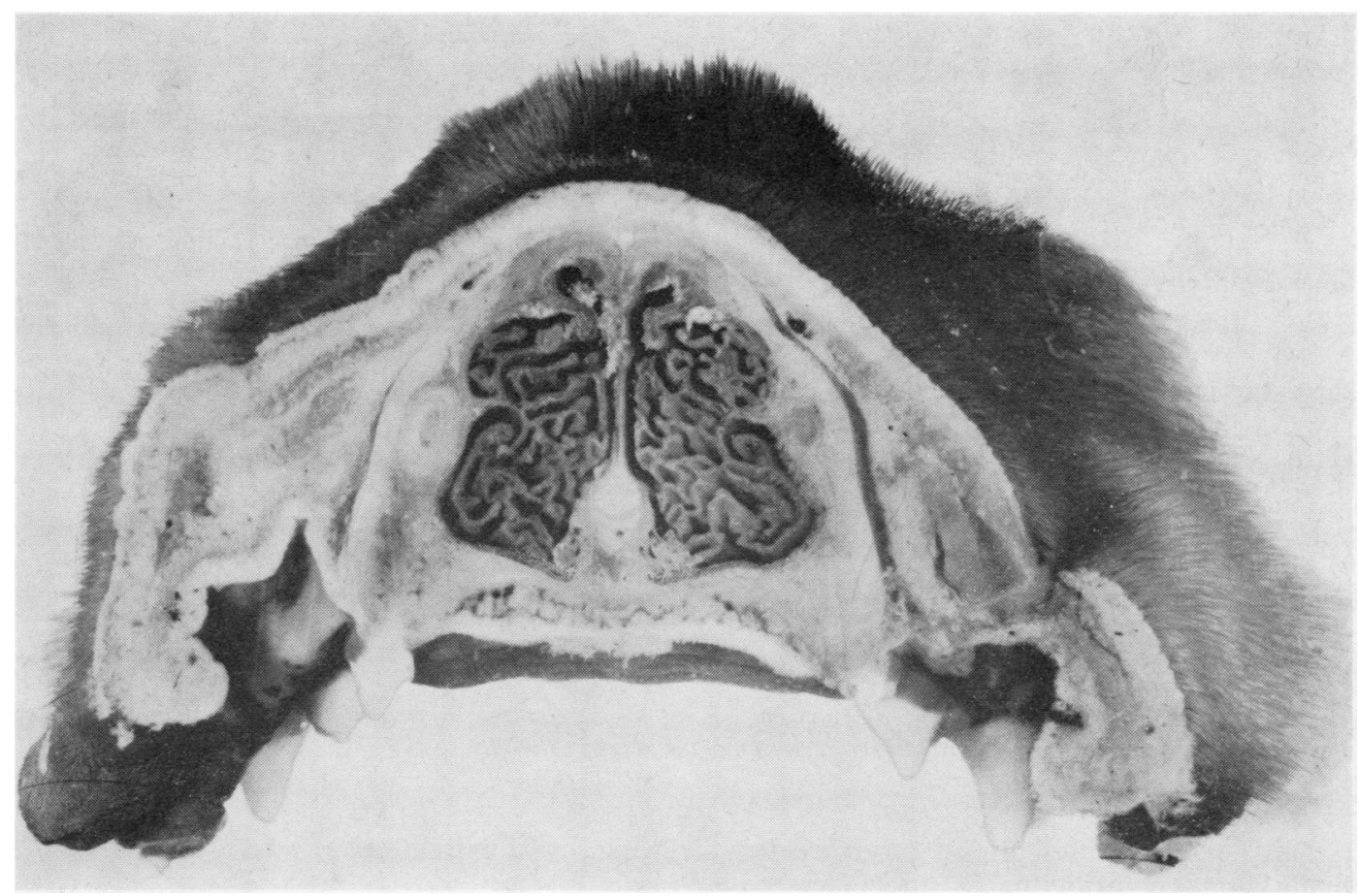

FIG. 2. Transverse section of the nose of a dog (Canis familiaris). The widely branching maxillo-turbinal bodies fill the snout. (Specimen E 155, Hunterian Museum R.C.S. Eng.)

\section{OBSTRUCTION AT THE NOSE}

The nose is primarily an olfactory organ, as evidenced by examination of species with varying degrees of olfactory acuity (Negus, 1964).

The nose of most animals is divided into two parts, anteriorly the maxillo-turbinal bodies with the function of warming and moistening inspired air, and posteriorly the ethmo-turbinal system carrying olfactory mucosa of varying extent (Fig. 3).

The size and complexity of the maxillo-turbinals determines the degree of obstruction in the nose ; thus in cats and dogs this body almost fills the lumen of the snout (Fig. 2), whilst in ungulates and in man the restricted turbinal area offers but slight resistance (Fig. 4).

Another consideration is the fact that man can breathe freely through the mouth if the nose is obstructed, but this is not so in most animals in which the epiglottis is in contact with the soft palate, thus shutting off the mouth as a possible portal for air. In higher apes and man the epiglottis does not reach the palate.

The degree of obstruction in the nose varies within wide limits by swelling or contraction of the mucosa, but not so rapidly as from one phase of respiration to another.

Beginning with the supposition that respiratory obstruction in most animals is greater than in man, it was decided to carry out investigations to determine the differences. The effect on the systemic

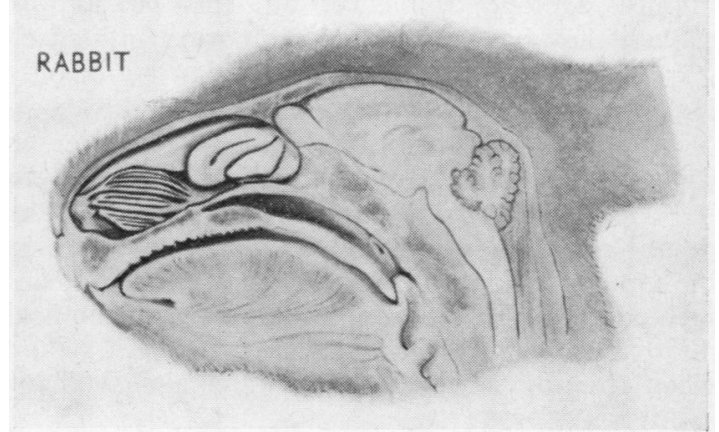

FIG. 3. Sagittal section of the head of a rabbit (Lepus cuniculus). Anteriorly the branching maxillo-turbinal is shown; posteriorly are placed the ethmo-turbinal bodies. The olfactory bulb is large. (Specimen E 145, Hunterian Museum R.C.S. Eng.) 

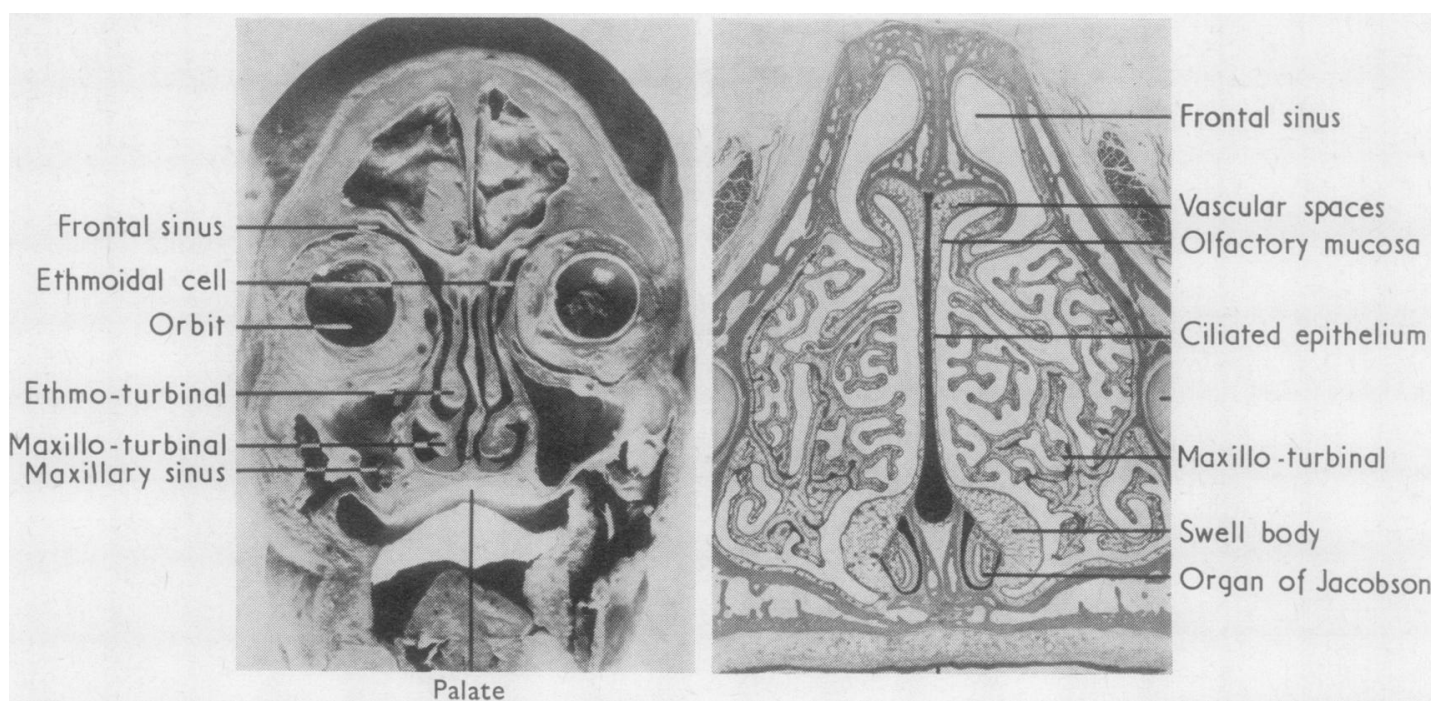

FIG. 4. Transverse sections of head of a man and snout of a cat for comparison of the restricted turbinals in the former and the elaborate branching of maxillo-turbinals in the latter.

and right heart pressures during nasal breathing. with the glottis contributing its quota, was compared with the influence of the glottis alone; and finally the intra-thoracic and intra-tracheal pressures were measured when glottic obstruction was eliminated by intubation or tracheostomy.

It was realized that no great effect would be found on the systemic arterial circulation because of the various compensatory mechanisms. In this respect Haldane (1922) wrote, 'The difference between arterial and venous pressures is so great that accessory factors such as the pumping movements of respiration can hardly have more than a very minute average influence on the circulation, though they have a marked temporary influence.'

In man, abnormal nasal obstruction is not only highly unpleasant but is also liable to lead to complications in the sinuses or the lungs. However, in spite of this, some explanation is required as to the considerable obstruction always present in the dog or cat, with no possibility of relief by opening the mouth. It might be argued from this that keenscented animals are at a considerable physiological disadvantage, but it is well recognized that a man when running is much slower than a cheetah or a dog.

The problems to be solved are the effects of the normal nasal obstruction of constant presence and rhythmic obstruction at the glottis. Furthermore, it must be discovered whether the considerable nasal obstruction of animals, especially in carni- vores, is of physiological advantage or disadvantage.

METHODS OF INVESTIGATION.

It will later be explained how measurements of pressure waves in the circulation were determined in man; here consideration is given to the phenomena in cats and dogs.

Anaesthesia was produced by nembutal, and fine catheters were then inserted into the femoral artery and vein; the catheters were passed to reach the arch of the aorta and the right atrium respectively. After death of the animal it was possible to verify that these positions were attained accurately.

A wide-bore needle was inserted into the trachea, and a catheter was passed through the mouth into the oesophagus to lie in its thoracic segment to measure intra-thoracic pressure.

Measurements of pressure changes in the oesophagus reflect changes in the thorax generally. There is a difference between this and intra-tracheal readings because in the former the recording tube has to be passed through the crico-pharyngeal sphincter, the function of which is to prevent inspired air from passing into the oesophagus instead of into the trachea (Negus, 1929a).

Air can enter the trachea more or less freely during both inspiration and expiration, depending on the degree of opening of the glottis; but it cannot enter the oesophagus because of the firmly closed cricopharyngeal sphincter. In consequence, changes of pressure shown by means of the tube inserted into the oesophagus do not change so readily as do those in 


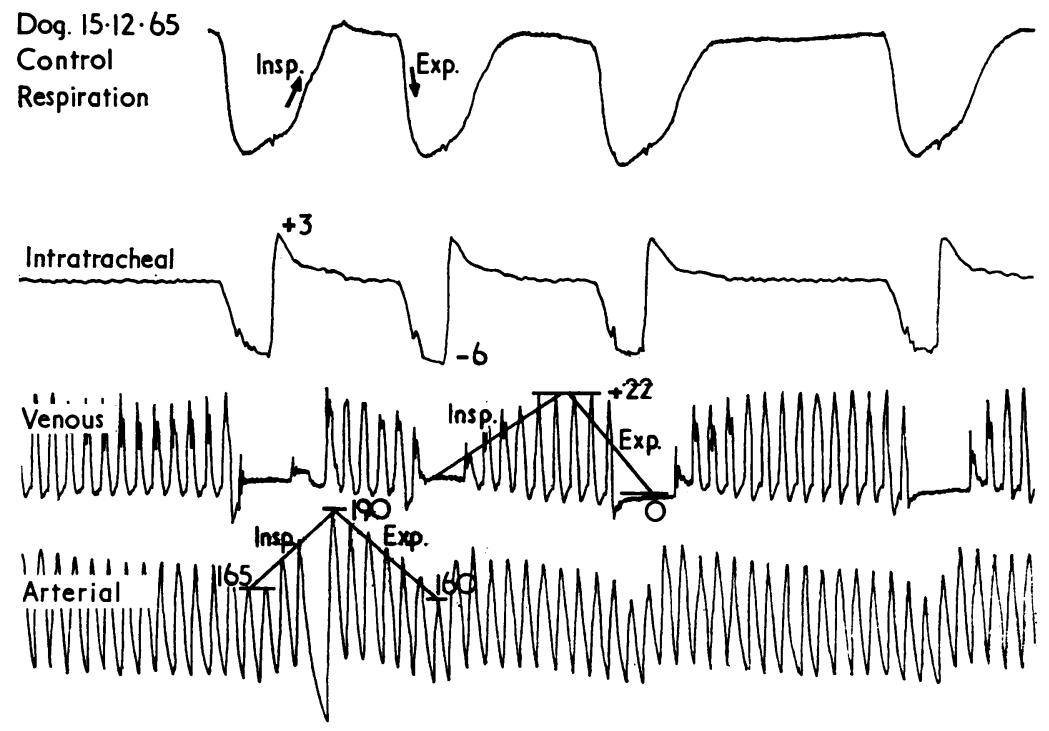

FIG. 5. Recording from a dog, mainly to show the changes of air pressure in the trachea varying with the phases of respiration. Respiratory waves in the venous and arterial systems are also shown.

the trachea. Intra-thoracic pressures were not recorded in human subjects. The phases of respiration were recorded by insertion of a small bag between the liver and the diaphragm, with upstroke during inspiration.

There were consequently five tracings to be recorded, namely the phases of respiration, and the intra-thoracic, intra-tracheal, venous, and arterial pressures. These were carried out by connecting the catheters and tubes to micro-manometers. The leads from the manometers were coupled to micro-galvanometers, from the mirrors of each of which a bead of light impinged on a strip of sensitive ruled paper, the range of movement of each being regulated by hand controls.

RECORDING OF RESISTANCE IN THE AIRWAY Figure 5 shows the changes in air pressure in the trachea of a dog during the phases of quiet respiration; the top curve records respiration, inspiration showing a rise in pressure as the intra-abdominal balloon is compressed. The next line is from the trachea; there is a rapid rise during inspiration terminating before the end of this phase and changing from a low pressure of $-6 \mathrm{~mm}$. $\mathrm{Hg}$ to a height of +3 , a total rise of $9 \mathrm{~mm}$. $\mathrm{Hg}$ or $117 \mathrm{~mm}$. $\mathrm{H}_{2} \mathrm{O}$.

This particular example shows a considerable degree of obstruction to the passage of air by the nose and glottis and its secondary effects on the circulation. Venous pressure is below atmospheric level during expiration, indicating the effect of considerable expiratory back-pressure on the thin-walled great veins and right atrium, rising at the beginning of inspiration to reach a level of $+22 \mathrm{~mm}$. $\mathrm{Hg}\left(286 \mathrm{~mm}\right.$. $\left.\mathrm{H}_{2} \mathrm{O}\right)$. The suction effect of inspiration draws blood into the right heart with an increase in pressure. There are large swings in time with the heart beats, but the respiratory waves are easily distinguishable.
The aortic circulation shows respiratory waves actually at a higher level than the venous but less in proportion. Rises due to each heart beat are $40 \mathrm{~mm}$. $\mathrm{Hg}$, while respiratory waves are $30 \mathrm{~mm}$. $\mathrm{Hg}$, the apex

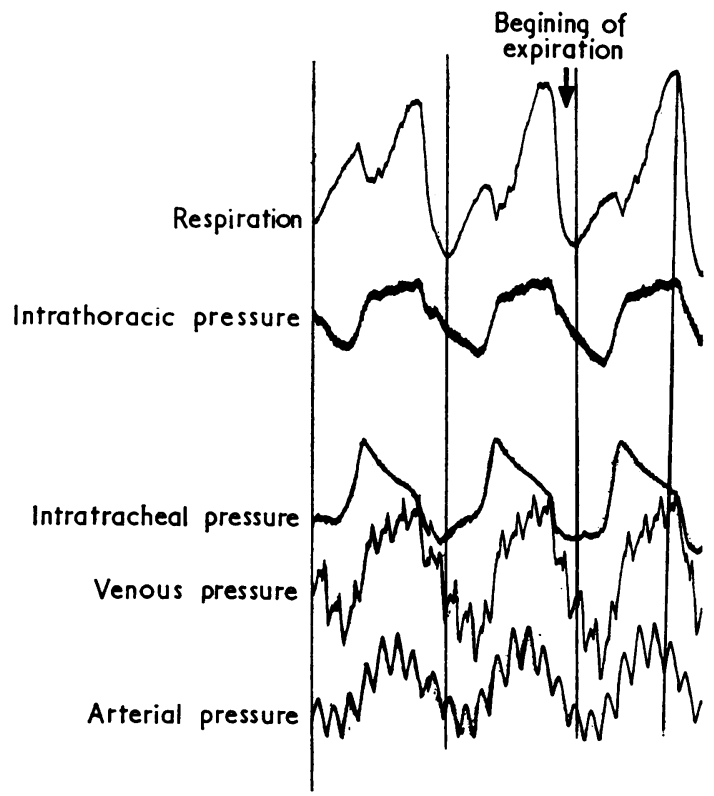

FIG. 6. Recording from a cat, with both nasal and glottic obstruction, showing the respiratory waves in the venous and arterial pressures rising during inspiration and dropping on expiration. The changes of intra-tracheal and intra-thoracic pressures are considerable. 
being $\quad 160-190 \mathrm{~mm}$. $\mathrm{Hg}$, illustrating Haldane's observation that the average influence on the circulation is not great.

Figure 6 is taken from an experiment on a cat and shows the effect of combined nasal and glottic obstruction; as in the dog, inspiration shows a rise of pressure and this is reflected in the intra-tracheal wave, when the increase starts at the beginning of inspiration but is completed long before the end of this phase.

In this experiment, intra-thoracic pressure is also recorded, but the timing is different from that of the intra-tracheal pressure, starting to rise well after the beginning of inspiration and completing its rise half way through that phase. In this tracing venous waves are marked and so are arterial variations; this aspect will be referred to later.

The conclusion from several observations is that the resistance offered to the passage of air by both nose and glottis is considerable; the effect on the venous circulation is marked, with a difference of between 18 and $28 \mathrm{~mm}$. $\mathrm{Hg}$ (156 or $286 \mathrm{~mm}$. $\mathrm{H}_{2} \mathrm{O}$ ).

Arterial pressure is less affected, the respiratory waves amounting to only a moderate percentage of the total.

INVESTIGATIONS ON HUMANS These were carried out by S. O. and D.C.B. on patients who were being investigated because of a systolic murmur. Only tracings from subjects who were proved subsequently to be normal have been considered. A needle was inserted into the trachea via the crico-thyroid membrane to record variations in pressure; respiratory movements were recorded by means of a thermistor held at the mouth and nose; arterial pressure was taken by a needle in the femoral artery, and venous changes by a catheter passed under fluoroscopic guidance from a vein in the right arm to lie in the right atrium. It will be seen (Fig. 7) that the tracings are similar to those in a dog (Fig. 5) or a cat (Fig. 6), but are of lesser amplitude, both in the trachea and in the venous and arterial circulations. This is due to the considerable obstruction offered by the nose in carnivores with its widely branching maxillo-turbinal bodies; even during deep respiration in the men examined the differences from carnivores were very obvious (Fig. 8, mean pressures; Fig 9, phasic pressures).

RESULTS OF REDUCED RESPIRATORY OBSTRUCTION AND RELATIVE EFFECT OF NASAL AND GLOTTIC OBSTRUCTION By passing a short laryngoscope in a cat or dog as far as, but not in contact with, the larynx, the glottis can be seen to be open, with widening during inspiration and narrowing during expiration.

The tube presses the soft palate against the posterior pharyngeal wall and detaches it from contact with the epiglottis. By this means air passes unhindered into the glottis and recording of the intra-tracheal pressure changes due to the glottis are obtained, the nasal resistance being eliminated.

The results from an experiment in a cat are shown in Fig. 10a, b. The tracheal pressure in Fig. 10b is -1 to $0 \mathrm{~mm}$. $\mathrm{Hg}$, compared with -3 to $+2 \mathrm{~mm}$. $\mathrm{Hg}$ in the control (Fig. 10a). The difference between $1 \mathrm{~mm}$. and $5 \mathrm{~mm}$., or a drop of $4 \mathrm{~mm}$., shows $80 \%$ to be

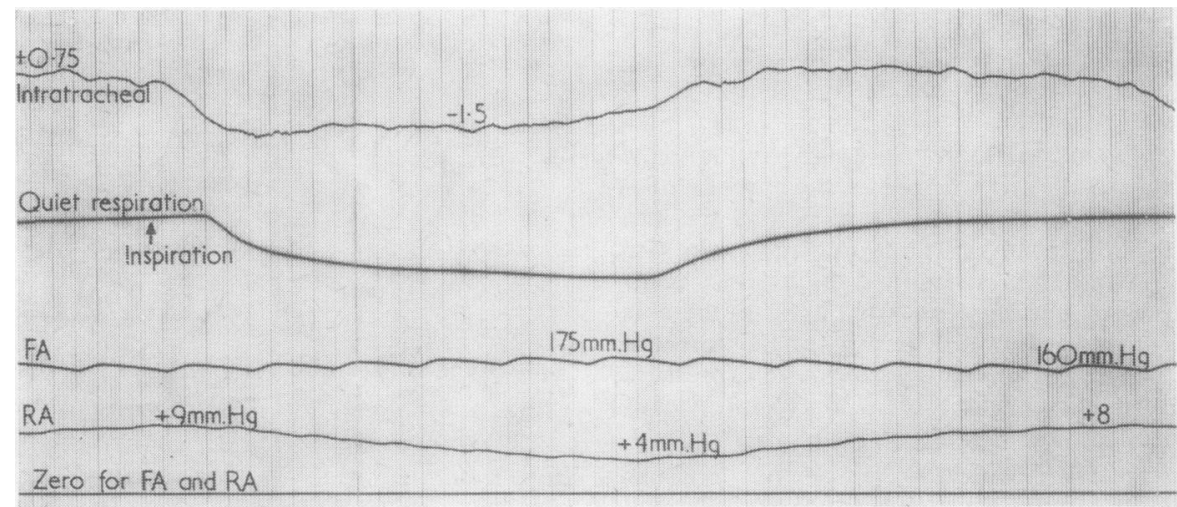

FIG. 7. Tracing from a man with no cardiac abnormality; with nose open and mouth closed during quiet respiration. The records are taken on mean instead of on phasic pressures to allow the swings to be more clearly seen.

The intra-tracheal pressure rises on inspiration and is lowered during expiration from $+0.75 \mathrm{~mm}$. $\mathrm{Hg}$ to $-1.5 \mathrm{~mm} . \mathrm{Hg}$, a swing of $1.8 \mathrm{~mm}$. $\mathrm{Hg}$. Pressure in the femoral artery shows only small changes with no obvious respiratory waves.

Right atrial (venous) pressure rises during inspiration to +8 or $9 \mathrm{~mm}$. $\mathrm{Hg}$. and is reduced to $+4 \mathrm{~mm}$. $\mathrm{Hg}$ during expiration; there are obvious respiratory waves of small extent.

All the changes of pressure are small compared to those in a dog (Fig. 5) or cat (Fig. 6). 


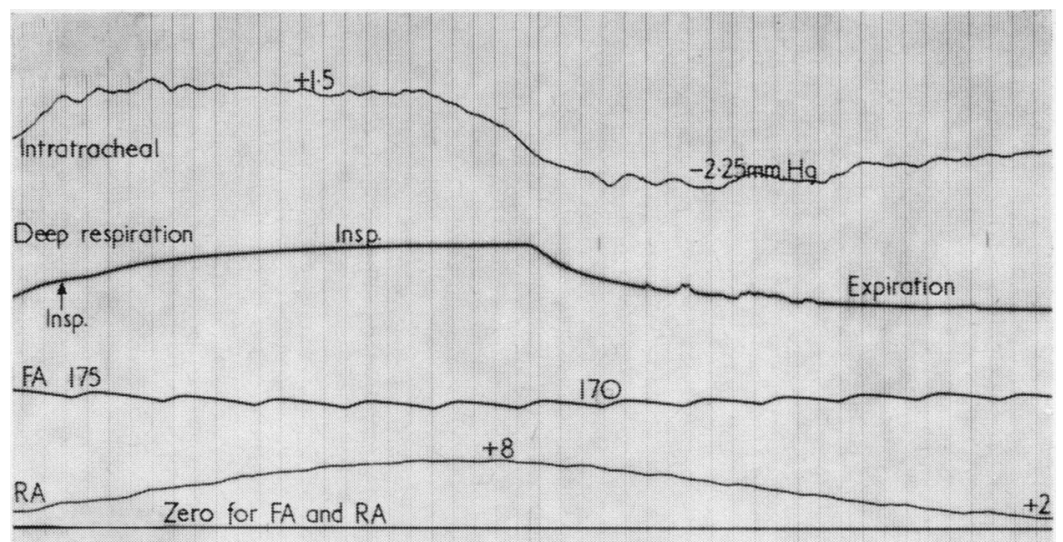

FIG. 8. Record from the same man as in Fig. 7 but during deep respiration with nose open and mouth closed, with intra-tracheal variations of $3.75 \mathrm{~mm}$. Hg. The arterial and venous changes of pressure do not vary materially from those in Fig. 7, although atrial pressure shows swings of $6 \mathrm{~mm} . \mathrm{Hg}$ instead of 4 $\mathrm{mm}$. Hg with quiet respiration. Comparison with Figs 5 and 6 illustrates the greater variation in carnivorous animals, where respiratory waves show venous swings of $22 \mathrm{~mm} . \mathrm{Hg}$ in a dog and ones of similar amplitude in a cat.

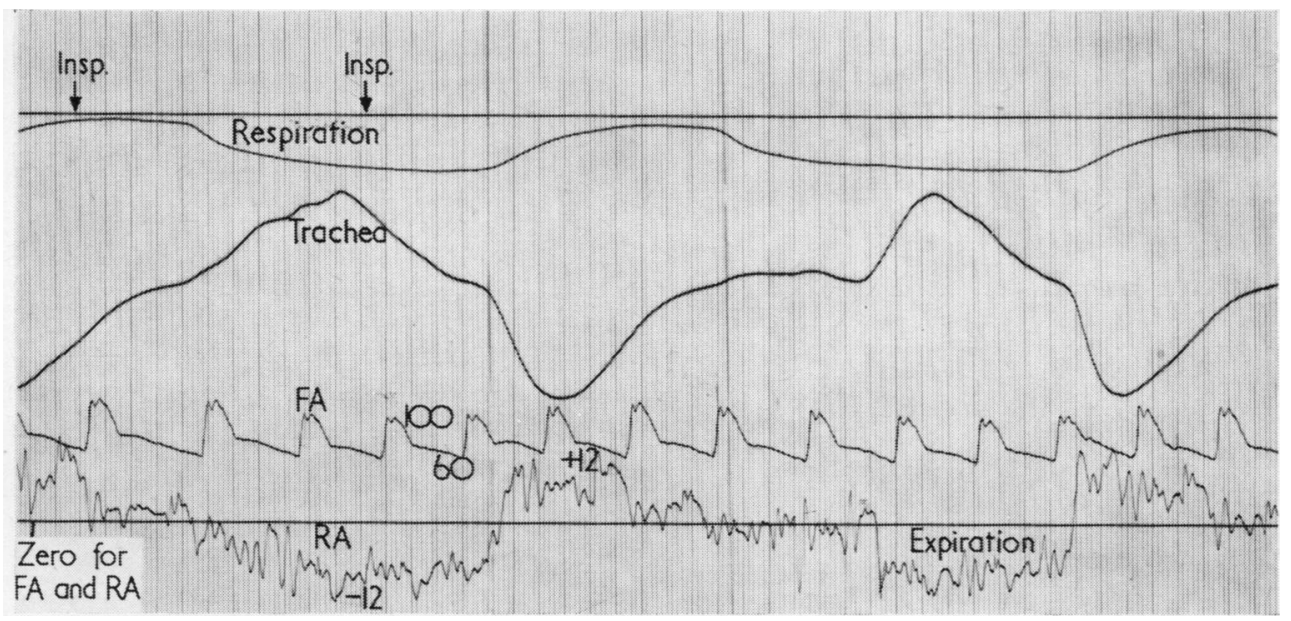

FIG. 9. From a different subject than the one in Fig. 8 but again with nose open and mouth closed during deep respiration.

The respiratory phases are well marked and the tracheal changes are very great compared with those in Fig. 8, which is also taken during deep respiration; there is no intratracheal calibration in this record.

The arterial changes are $100 \mathrm{~mm} . \mathrm{Hg}$ and $60 \mathrm{~mm} . \mathrm{Hg}$ during systole and diastole, but there are no measurable respiratory waves.

The venous waves are well marked from $+12 \mathrm{~mm}$. $\mathrm{Hg}$ to -12 , a swing of $24 \mathrm{~mm}$. $\mathrm{Hg}$, comparable to those in a dog during quiet respiration; in this recording (Fig. 5) the respiratory movements are greater than in man. 


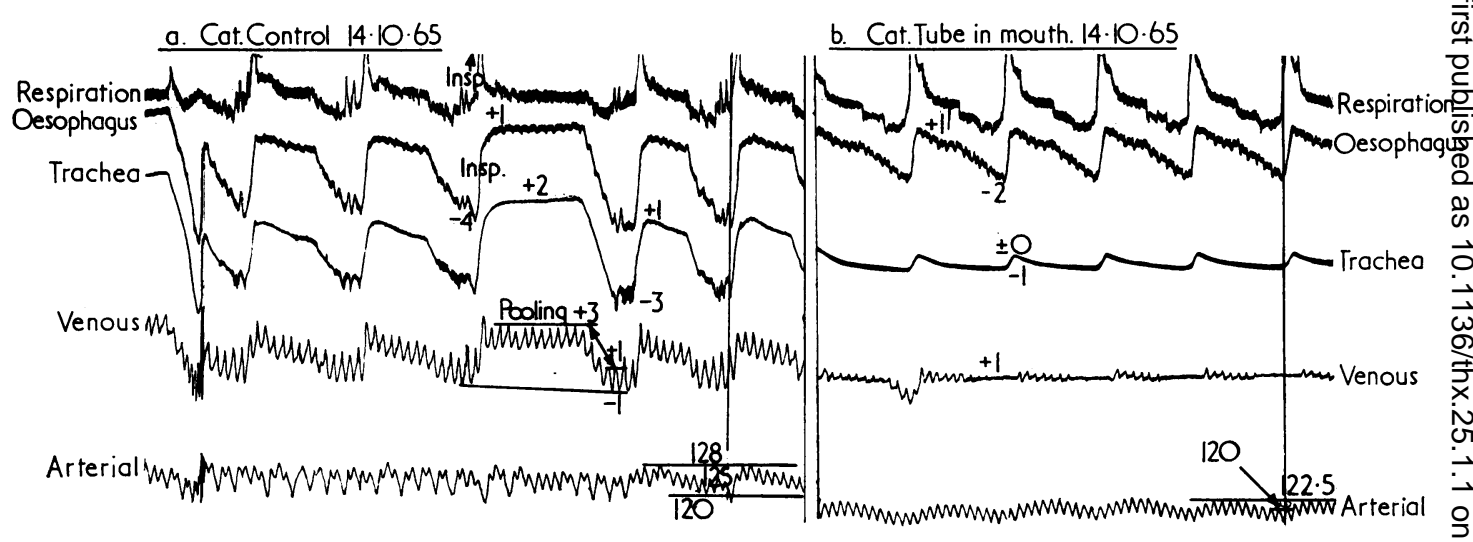

FIG. 10. (a) Control recording from a cat with considerable changes of intra-tracheal and intra-thoractic pressures during quiet respiration. (b) Recording from the same cat when nasal resistance has been eliminated 年y the passage of a wide-bore tube into the trachea.

attributable to the nasal resistance and $20 \%$ to that at the glottis and in the bronchi.

The intra-thoracic pressures vary to a lesser degree, $3 \mathrm{~mm}$. $\mathrm{Hg}$ with the nose eliminated (Fig. 10b) and $5 \mathrm{~mm}$. Hg with it included (Fig. 10a), a drop of nearly $50 \%$. Venous pressures show only slight variations and arterial changes are also slight.

Another experiment showed a considerable tracheal pressure in the control and a much lower one when a tube was led down to the glottis.

In man there is no necessity for a tube in the mouth, because air can pass freely into the trachea when the mouth is open. One experiment showed $\overrightarrow{9}$ low intra-tracheal pressure change of $3 \mathrm{~mm}$. $\mathrm{Hg}$, क negligible variation in arterial and a very small change of $3 \mathrm{~mm} . \mathrm{Hg}$ in the right atrial pressure, more or lese in correspondence with our findings in a cat after tracheostomy.

With deep breathing in man, another experimen showed the effects of respiratory resistance to b\& more marked, due partly to the relative narrowness of the glottis in relation to the calibre of the trache and also to the friction in the smaller air passages, but the vascular changes are slight.

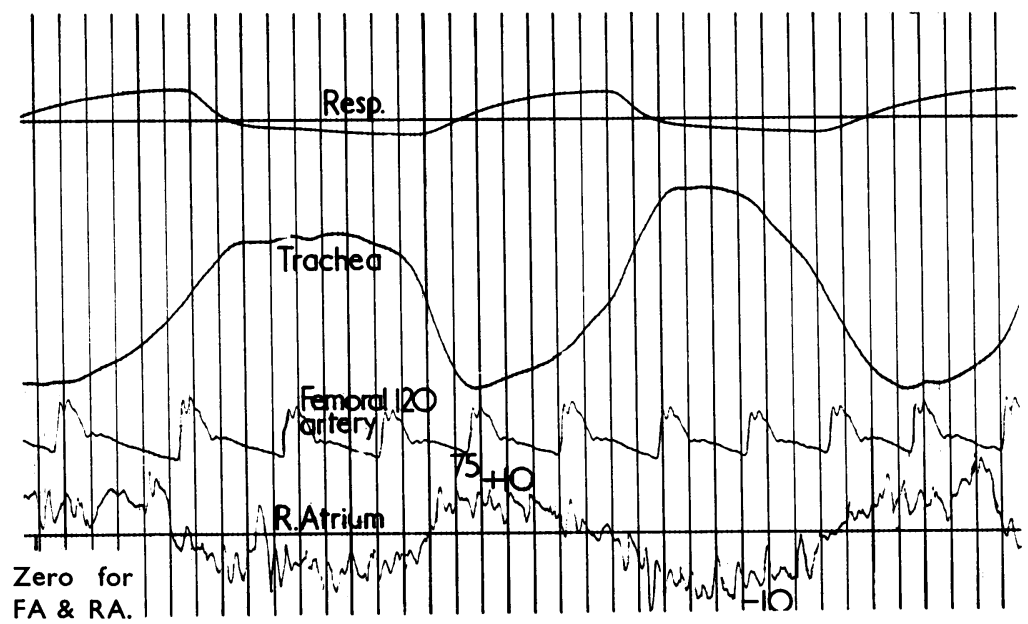

FIG. 11. Recording from a man with the right nostril and the mouth closed. The tracheal variations were big but were not calibrated. The arterial pressure was $120 / 75 \mathrm{~mm} . \mathrm{Hg}$ and the venous fluctuations were $+10 \mathrm{~mm} . \mathrm{Hg}$ to $-10 \mathrm{~mm} . \mathrm{Hg}$, a swing of $20 \mathrm{~mm}$. Hg. All pressures were phasic. 
RESULT OF ELIMINATION OF GLOTTIC RESISTANCE By performing tracheostomy or by inserting a sufficiently wide-bore tube into the trachea the resistance at the glottis is eliminated.

In this experiment it was seen that intra-tracheal pressure changes were eliminated, with a corresponding reduction of the venous curve; arterial waves were also slight as compared with the changes caused by nasal and glottic obstructions combined.

Venous and arterial waves were slight in comparison with those in the control animal, especially the arterial.

EFFECTS OF ABNORMAL INCREASE IN RESPIRATORY OBSTRUCTION Closing one nostril of a cat or man reduces air intake and output very considerably. The intra-tracheal changes in pressure increase markedly during inspiration and expiration. Intra-thoracic variations are also increased, mainly on the side of subatmospheric pressure, movement of the chest wall and diaphragm having greater difficulty in drawing air into the lungs and expelling it (Figs 11 and 12). Both venous and arterial respiratory waves are only slightly affected when one nostril is closed, which is surprising.

\section{CONCLUSIONS}

The early opinion that pump action on the circulation was due mainly to obstruction at the glottis

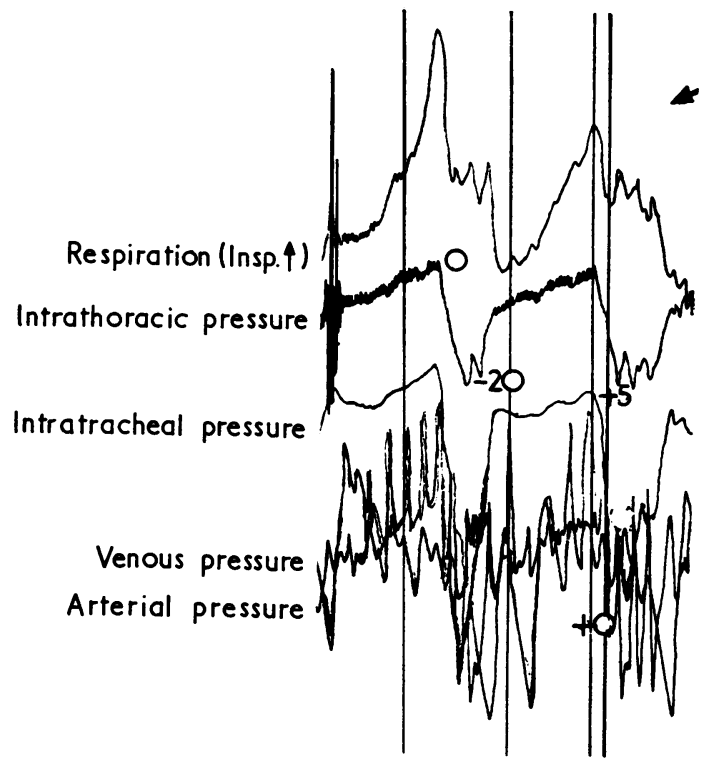

FIG. 12. Closing one nostril in a cat raises the intrathoracic pressure slightly, and intra-tracheal pressure considerably, as compared with the control. Venous and arterial waves are increased but overlapping confuses the record. is incorrect ; the main point of resistance is in the nose, which offers constant resistance, variable in wide periods of time but not so rapidly as the phases of respiration.

The second observation is that with elimination of nasal resistance there is still some obstruction at the glottis of cats and dogs, varying with inspiration and expiration; it appears that the glottis is the point of fine adjustment.

The normal resistance at the nose and glottis has a considerable effect on both intra-tracheal and intra-thoracic pressures and these changes are transmitted to the venous circulation, exerting a suction effect on the great veins and right atrium, with dilatation of pulmonary capillaries and pooling in the lungs.

The physiological effect would appear to be more efficient respiratory exchange between alveoli and pulmonary capillaries, increased when there is an ample supply of blood to meet increased aeration, thus helping to keep arterial $\mathrm{CO}_{2}$ content constant.

Respiratory waves in the arterial circulation are present, but they are slight in proportion to the high systemic pressure, being no more than 11 or $16 \%$ of the total, in accordance with Haldane's statement.

But, even if arterial output is not increased materially, it appears likely that all the blood reaching the left side of the heart is adequately oxygenated, with constant percentage of $\mathrm{CO}_{2}$ and with obvious benefit to the tissues receiving arterial blood. Even if not a primary function of pump action, this may be a fortunate and useful result; it would be more marked in animals with an obstructed nose than in man and apes.

In both carnivores and man the altering pressure on the great veins and right side of the heart, especially the atrium, encourages the onward flow of blood during the inspiratory phase and obstructs the flow during expiration; obvious waves of increase and reduction, in time with the waves of respiration, can be seen in man and more so in animals. This is due mainly to the obstructed nature of the nose with extensive maxillo-turbinal bodies, and is most marked in the keen scented species ; man with his relatively feeble powers of scent has a nose of much freer lumen and consequently with less obstruction.

The glottis also has an effect, but of a lesser degree, and the many-branched bronchi and bronchioles again cause obstruction by friction at the numerous junctions.

Investigations support Haldane's opinion (1922) that 'breathing respresents the lung ventilation 
required to keep the reaction and pressure of oxygen in the blood supplying the respiratory centre constant within certain narrow limits. . . . It is now realized that the pressure of $\mathrm{CO}_{2}$ is as important too.

The varying flow of blood from the right side of the heart is obvious in experiments on animals and man, but the left side of the heart is not similarly influenced, as Haldane (1922) observed: 'The difference between arterial and venous pressures is so great that accessory factors such as the pumping movements of respiration can hardly have more than a very minute average influence on the circulation, though they may have a marked temporary influence.'

In regard to the relative merits or disadvantages of upper respiratory obstruction in animals or man the conclusion is reached that slight retardation of air entry, added to the suction effect on the great veins and pulmonary capillaries during inspiration, has no deleterious effect on arterial circulation and may on the contrary give benefit in maintaining the correct percentage of $\mathrm{CO}_{2}^{\stackrel{\vec{F}}{\vec{*}}}$ regulating the respiration and maintaining an adequate supply of oxygen.

Expiration, on the other hand, conserves energy in retarding the passage of blood through the lungso when insufficient fresh air is in the alveoli for oxygenation and exchange of carbon dioxide.

To Professor David Slome we offer gratitude for facilities in his Department of Physiology at the Royalo College of Surgeons, and for the help there of $\mathrm{Mr}: \overrightarrow{\vec{x}}$ L. S. Drewell and Mr. L. Cavanagh. We wish also to thank Mr. D. Kitchen, of the Physics Department off King's College Hospital, for his expert technical help.-

\section{REFERENCES}

Haldane, J. S. (1922). Respiration. Yale University Press, New Haven Negus, V. E. (1929a). The function of the crico-pharyngeus muscle In First International Congress of Oto-rhino-laryngology, Copen hagen, 1928, p. 575.

(1929b). The Mechanism of the Larynx. Heinemann, London. (1958). The Comparative Anatomy and Physiology of the Nose and Paranasal Sinuses. Livingstone, Edinburgh. (1964). The primary function of the nose. Int. Rhinol., 2, 